\title{
A Tool for Interactive Disease Outbreak Visualization, Detection, and Forecasting
}

\author{
Jarad Niemi* \\ lowa State University, Ames, IA, USA
}

\section{Objective}

To build a zero-cost tool for disease outbreak visualization, detection, and forecasting incorporating modern tools.

\section{Introduction}

Interactive tools for visualization of disease outbreaks has been improving markedly in the past few years. With the flagships Google Flutrends ${ }^{1}$ and HealthMap ${ }^{2}$ providing prime examples. These tools provide interactive access to the general public concerning the current state-of-affairs for disease outbreaks generally and specifically for influenza. For example, while browsing HealthMap I learned of a case of tuberculosis on my campus, Iowa State University. While extremely sophisticated, these tools do not utilize modern statistical algorithms for detection or forecasting. In addition, the development cost and perhaps the maintenance cost is not trivial.

We aim to build a similar visualization tool that incorporates modern algorithms for detection and forecasting but has low development and maintenance cost. Due to the low cost this tool is appropriate for quick deployment in developing countries for emerging outbreaks as well as public health agencies with declining operating budgets.

\section{Methods}

The tool of choice for development of this low-cost visualization tool is the R statistical environment ${ }^{3}$ and the shiny ${ }^{4}$ package. Use of $\mathrm{R}$ and relevant package incurs zero cost for the software itself and allows access to any algorithms currently implemented in any R package or script. The shiny package provides a framework for constructing the tool where code is written entirely in R, but the deployment of the tool is in HTML which can be run locally or globally on the web.

\section{Results}

A demonstration tool has been created using a 2010 measles outbreak in Zimbabwe as an example case. To our knowledge, the data collected during this outbreak was solely available in pdf files which limits the ability to visualize the data for the purpose of public health policy decision making. In less than 100 lines of pure R code, a visualization tool has been created that allows a user to select which districts to visualize and whether to aggregate the new cases across districts. A plot of the new cases by week is then immediately updated as the user selects or unselects districts as well as whether to aggregate those districts.

\section{Conclusions}

A primary motivation for presentation at this conference is to solicit feedback from public health practitioners for the design specifications for such a tool and how it could best be utilized for public health decision making. The options for such a tool are 1) to aid in data collection [which might be particularly a concern in developing countries without a existing standardized procedures], 2) detection of outbreaks using modern algorithms including spatio-temporal detection, 3) vizualization of existing data, and 4) forecasting disease severity and duration.

\section{Measles Outbreak in Zimbabwe}

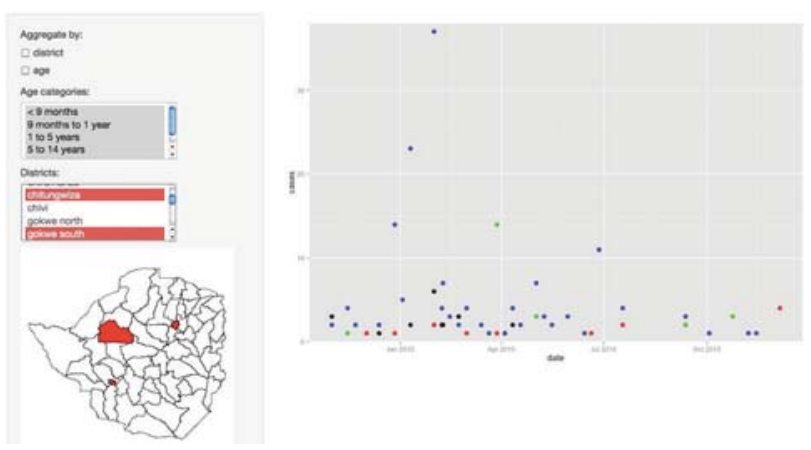

\section{Keywords}

interactive visualization; disease detection; forecasting; R; shiny

\section{References}

[1] Ginsberg, Jeremy, Matthew H. Mohebbi, Rajan S. Patel, Lynnette Brammer, Mark S. Smolinski, and Larry Brilliant. "Detecting influenza epidemics using search engine query data." Nature 457, no. 7232 (2008): 1012-1014

[2] Freifeld, Clark C., Kenneth D. Mandl, Ben Y. Reis, and John S. Brownstein. "HealthMap: global infectious disease monitoring through automated classification and visualization of Internet media reports." Journal of the American Medical Informatics Association 15, no. 2 (2008): 150-157.

[3] R Core Team (2013). R: A language and environment for statistical computing. R Foundation for Statistical Computing, Vienna, Austria. http://www.R-project.org/.

[4] RStudio and Inc. (2013). shiny: Web Application Framework for R. $\mathrm{R}$ package version 0.7.0. http://CRAN.R-project.org/package=shiny

\section{*Jarad Niemi}

E-mail: niemi@iastate.edu 